De Tampere à Séville : bilan de la sécurité européenne $(1 / 2)$

\title{
Logiques et pratiques de l'Etat délégateur : les compagnies de transport dans le contrôle migratoire à distance. Partie 2
}

\section{Virginie Guiraudon}

\section{(2) OpenEdition \\ Journals}

\section{Édition électronique}

URL : http://journals.openedition.org/conflits/773

DOI : $10.4000 /$ conflits. 773

ISSN : $1777-5345$

Éditeur :

CCLS - Centre d'études sur les conflits lilberté et sécurité, L'Harmattan

Édition imprimée

Date de publication : 1 mars 2002

ISBN : 2-7475-3029-9

ISSN : 1157-996X

Référence électronique

Virginie Guiraudon, «Logiques et pratiques de l'Etat délégateur : les compagnies de transport dans le contrôle migratoire à distance. Partie 2 », Cultures \& Conflits [En ligne], 45 | printemps 2002, mis en ligne le 22 mars 2006, consulté le 30 mars 2021. URL : http://journals.openedition.org/conflits/773 ; DOI : https://doi.org/10.4000/conflits. 773

Ce document a été généré automatiquement le 30 mars 2021.

Creative Commons License 


\title{
Logiques et pratiques de l'Etat délégateur : les compagnies de transport dans le contrôle migratoire à distance. Partie 2
}

\author{
Virginie Guiraudon
}

L'attitude des compagnies de transport

Les responsables des compagnies aériennes n'aiment pas parler de leur rôle de «sheriff's deputy». Il est difficile d'obtenir des entretiens et des informations concrètes : une réalité que les autres chercheurs qui se sont aventurés sur ce terrain (Antonio Cruz, Kristeen le Bourhis, Gallya Lahav) ne manquent pas de souligner mais également le personnel des entreprises. Les raisons invoquées par les compagnies varient : procédures judiciaires en cours, mauvaise image de marque pour l'entreprise, accord confidentiel avec le gouvernement sur les objectifs à atteindre. Les autorités elles-mêmes n'ont que rarement et très sommairement procédé à une évaluation de ces politiques. Ainsi en France il faut remonter à 1996 pour avoir des chiffres officiels et partiels dans le rapport au Parlement.

Il existe également d'énormes variations selon les compagnies. Les compagnies qui coopèrent le plus avec les autorités sont les plus silencieuses. Celles qui comme British Airways sont à la fois les plus exposées aux sanctions car les passeurs empruntent leurs nombreuses lignes $^{1}$ et qui se rebiffent contre la mise en œuvre du système des sanctions sont plus loquaces. D'autres facteurs entrent en jeu. KLM a beaucoup été sanctionnée ${ }^{2}$. KLM a perdu en appel devant la Cour suprême et doit payer plusieurs millions de florins. En attendant la décision, elle avait voulu montrer son esprit de coopération en signant un protocole d'entente avec l'IND (le département chargé de l'immigration au ministère de la Justice) et la maréchaussée néerlandaise. Elle adopte donc un profil bas pour faciliter les rapports avec les autorités tout en évitant toute publicité sur ces sujets. Il en va de même pour les autres compagnies comme Lufthansa qui ont signé des accords d'entente (au Canada) et parce que l'Allemagne a des lois 
assez strictes. Pour certains, des procès sont en cours. Leur silence est gêné car la coopération se fait mais sans qu'elles puissent en être fières.

L'alliance entre gouvernements et compagnies aériennes est formalisée dans le cas canadien, belge, allemand ou néerlandais des protocoles d'entente mais existe ailleurs. Aux Etats-Unis, le Immigration and Nationality Technical Corrections Act de 1994 a annulé pour bons résultats ("good performance ») les amendes des compagnies aériennes qui pouvaient montrer qu'elles avaient contrôlé ses passagers selon la législation en vigueur. En outre, si les compagnies participent au "Approved Gate Check system », elles peuvent bénéficier d'exemptions pour les amendes. Logique d'Etat, logique de marché ? La façon dont les agences de l'Etat marchandent avec les compagnies montre que la réponse à cette question n'est pas si claire. En effet, chaque protocole d'entente fait l'objet de négociations entre l'Etat et l'entreprise sur les objectifs de rendement et sur les compensations à verser si les objectifs ne sont pas atteints.

Le flou entre privé et public domine également sur le terrain dans les aéroports de départ désignés sous le nom de "hot spots » ou «points chauds », en particulier dans les aéroports africains. Les contrôles de documents au départ sont assurés par des sociétés de sécurité locales sous-traitantes depuis longtemps ${ }^{3}$. Mais il y a aussi des fonctionnaires en détachement qui aident des équipes. Au tout début de la mise en œuvre des sanctions contre les transporteurs et du durcissement du droit d'asile, ce procédé était plutôt artisanal. Ainsi, en 1990, des officiers de la police fédérale des frontières allemandes étaient "déguisés " en personnel des compagnies aériennes assurant des vols vers l'Allemagne à partir des points chauds en l'absence d'accords bilatéraux avec les pays d'origine ${ }^{4}$.

Cette technique s'est généralisée mais surtout elle figure parmi les objectifs affichés et non plus déguisés du Conseil des ministres «Justice et Affaires intérieures » de l'Union européenne depuis au moins 1996. En octobre en effet, les ministres ont décidé d'organiser des missions communes d'assistance et d'information par des officiers y compris auprès des personnels des compagnies responsables des contrôles de documents ${ }^{5}$. La position commune les décrivait ainsi : les missions d'assistance «ont pour but d'assister les agents localement chargés des contrôles soit pour le compte des autorités locales, soit pour le compte des compagnies aériennes» et les «missions communes d'information des personnels des compagnies aériennes [...] ont notamment pour objet de décrire les documents et visas requis par les Etats membres ainsi que les modalités de vérification de la régularité de ces documents et visas ».

Dans le cadre de Schengen puis de l'Union européenne, de nombreux officiers de liaison ont été envoyés dans les pays d'origine et de transit. Parmi leurs missions figurent le contrôle à l'embarquement et l'assistance aux compagnies aériennes. Le plan d'action pour améliorer le contrôle de l'immigration proposé par la Présidence française le 4 juillet 2000 avec le soutien officiel de l'Allemagne et l'Italie prévoit comme mesure le renforcement de la coopération entre officiers de liaison affectés aux compagnies dont la mission est décrite comme un "système[s] permanent[s]». En outre, afin de mutualiser les ressources, il préconise qu'en fonction des connections aériennes avec les pays de destination, un officier de liaison se concentre uniquement sur le « contrôle à l'embarquement » mais pour les vols à destination de tous les pays de l'Union et non seulement du sien 6 .

Ainsi si les sanctions contre les transporteurs servent à obliger les compagnies à améliorer le contrôle des documents, elle justifie aussi l'envoi de fonctionnaires pour 
leur permettre de mieux remplir leurs obligations. Au final, il y a plus d'officiers sur place avant qu'après les lois ${ }^{7}$. Le ministre français Daniel Vaillant a déclaré après l'adoption de la directive européenne sur les sanctions contre les transporteurs qu'il fallait « renforcer, sur place, la coopération policière avec le pays d'origine ou de transit. Malgré les actions et les efforts entrepris, force est pourtant de constater que les contrôles effectués par les compagnies aériennes et les services de police aux aéroports demeurent insuffisants, particulièrement en Afrique subsaharienne $»^{8}$.

Parmi les employés chargés de la mise en place de la vérification des documents dans les compagnies de transport ou de ceux des entreprises de sécurité sous-traitées, on retrouve des anciens fonctionnaires de la police de l'air et des frontières, des douanes, des services de renseignement, ou de la police. C'est cependant Eurotunnel qui a assuré la reconversion la plus étonnante en nommant en juillet 2001 un général britannique de l'armée de terre à la retraite, Sir Roger Wheeler, pour améliorer la sécurité afin d'empêcher les étrangers de Sangatte d'atteindre le Royaume-Uni' .

Les agences de sécurité comme AVS en Allemagne qui est chargé du contrôle des documents à Francfort recrutent essentiellement d'anciens policiers et d'anciens de la Bundesgrendschutz. Le directeur d'AVS n'est autre que l'ancien chef de la sécurité de Francfort. En France, le responsable de la sécurité à Air France, M. Catala, est un fonctionnaire de l'Intérieur en détachement avec le grade de Commissaire. Avant de prendre ses fonctions à Air France, il était en poste à la Police de l'Air et des Frontières à Pau avant de rejoindre Roissy au même poste. Le responsable de KLM, M. Platenkamp, a occupé des fonctions importantes dans la police à Amsterdam avant de travailler dans un bureau de lutte anti-terroriste (service central d'information des enquêtes) et a ainsi effectué un stage avec le FBI aux Etats-Unis. Il est d'ailleurs reconnu comme un spécialiste du terrorisme et intervient sur ces questions dans les colloques internationaux et les groupes de travail de l'IATA ${ }^{10}$. Dans certains cas, les personnes en question ont été "démissionnées » du secteur public avant de se reconvertir dans le privé. Il faut noter que les compétences des responsables de la vérification des documents ne sont pas issues d'une expérience ou d'une formation dans le domaine de l'immigration mais de la sécurité au sens large et dans de nombreux cas, la question des contrôles de documents est traitée par la direction de la sécurité. On peut comparer cette situation à la gestion des problèmes de l'immigration dans le cadre de Schengen puis du Troisième Pilier de l'UE. Là aussi l'immigration figurait parmi d'autres domaines d'intervention tels que le terrorisme, le trafic de drogues ou d'armes, etc.

Deuxièmement, il semble que les services au sein des entreprises de transport aérien chargés de la sécurité ont une place importante dans l'organigramme de l'entreprise. Ils sont généralement directement rattachés à la direction (comme à Air France) ou bien le responsable siège au comité directeur (le cas de KLM). Ils ont donc une voix importante même si leurs objectifs peuvent rentrer en conflit avec ceux d'autres directions telle que la direction des relations internationales qui voudraient avoir de bonnes relations avec les pays d'origine, comme certains responsables de ces directions nous l'ont $\operatorname{dit}^{11}$. Ces conflits internes à l'entreprise reproduisent des clivages interministériels entre Intérieur et Affaires Etrangères ou inter-piliers dans l'Union européenne, comme si en somme on avait déplacé dans le privé le jeu des luttes d'influence du domaine public.

L'argumentation des responsables de la sécurité au sein de l'entreprise diverge du discours public de dénonciation des lois imposant des sanctions. Elle repose sur ces lois 
au contraire pour dire qu'on ne peut pas ne pas les appliquer et faire l'impasse sur les contrôles. Le discours des responsables interrogés reprend aussi à son compte une partie du discours sécuritaire sur l'immigration en présentant les migrants potentiels comme toujours plus nombreux et désespérés.

Ainsi se met en place une dynamique d'institutionnalisation des problèmes mis sur l'agenda. Elle ressemble à celle qui a vu la montée en puissance des services de ressources humaines aux Etats-Unis en réponse à la complexité de la législation à laquelle les entreprises devaient se mettre en conformité et qui a donc entraîné la création de services spécialisés qui se sont efforcés de démontrer aux dirigeants l'importance de la législation pour assurer leur pérennité dans l'entreprise ${ }^{12}$.

Dans le cas des services de sécurité, on voit également ce processus de pérennisation, de professionnalisation et d'autonomisation qui leur permet de devenir une institution au sein de l'organisation privée. En France par exemple, les premiers contrôleurs de documents d'Air France à Roissy étaient des contrôleurs commerciaux reconvertis, puis on a formé le personnel au sol avant de créer un groupe spécialisé d'une trentaine de personnes qui se tient à côté des personnels d'enregistrement et vérifie les documents de voyage (après une formation de quelques jours). Les autres contrôles avant l'embarquement sont sous-traités. Cette sous-délégation à d'autres opérateurs privés augmente le nombre d'acteurs pour qui les lois imposant des sanctions ne représentent pas un coût mais une aubaine.

Ce que l'on observe au sein des entreprises et au niveau des politiques de contrôle migratoire a son pendant au sein des instances professionnelles internationales, notamment IATA (International Air Transport Association) et ICAO (International Civil Aviation Organization) et l'ECAC (European Civil Aviation Conference) ${ }^{13}$. Certains responsables de la sécurité des compagnies aériennes rencontrés occupent des fonctions importantes dans IATA et font partie des groupes de travail liés aux questions de sécurité ${ }^{14}$ tel que le Control Authorities Working Group (CAWG). IATA organise des formations de personnel sur les contrôles des documents de voyage. ICAO comporte aussi des groupes spécialisés tels que le New Technology Working Group qui travaille sur l'application de la biométrique et des technologies visant à lire en machine les passeports et les visas à la détection des «non-admis potentiels ».

Un exemple récent de la légitimité que ces organisations confèrent à la thématique du contrôle migratoire concerne la tenue d'une conférence organisée par IATA à Atlanta le 15-16 octobre 2001 intitulée «Human Traffic: responding to the threat ... to protect your interests » dont le sous-titre ne parlait d'ailleurs pas de trafic mais d'immigration illégale: "Human traffic will assess the political and financial consequences of illegal migration. Airlines and receiving nations will examine solutions to successfully combat this illicit trade ». Pour un peu plus de $\$ 800$, on pouvait écouter pêle-mêle des représentants des services de l'immigration, des compagnies aériennes, des entreprises de sécurité, de la Commission européenne (Jean-Louis de Brouwer, DG Justice et Affaires intérieures).

Cette conférence était sponsorisée par plusieurs entreprises spécialisées dans les technologies de contrôle (documents lisibles en machine, biométrique, banque de données) telles que Imaging Automation, ICTS International NV, IDsystems ou Renful aviation security limited. Les deux premières ont également présenté leurs solutions au problème de l'immigration illégale dans la rubrique "solutions commerciales». Les sommes en jeu sont ici considérables: les contrats sont importants et ces entreprises 
cotées en bourse font des chiffres d'affaire toujours plus élevés. Comme les anciens fonctionnaires qui travaillent pour les compagnies, il s'agit pour elles d'adapter au contrôle migratoire le "savoir faire " acquis dans des domaines aussi divers que le traitement des feuilles maladie dans le New Jersey, les cartes d'identité argentines ou la lutte anti-terroriste dans les aéroports. Imaging Automation a d'ailleurs déjà de l'expérience en la matière, ayant été choisie par la République dominicaine pour sécuriser ses frontières et aéroports.

On voit ainsi que le paysage est plus complexe qu'une simple délégation d'une fonction régalienne au secteur privé. Les entreprises de technologie de la sécurité travaillent indifféremment pour des Etats ou des compagnies aériennes et peuvent d'ailleurs employer comme consultants des responsables de ces derniers. Elles sont partenaires de l'IATA, financent des conférences sur la sécurité aérienne, et dans le cadre de la concurrence commerciale peuvent offrir des "packages » aux compagnies qui incluent le contrôle des documents. D'un autre côté, les compagnies aériennes peuvent investir dans ces compagnies aux bénéfices alléchants.

Un dernier point a trait à la redéfinition du débat sur l'immigration induit par l'entrée en scène des transporteurs. Les arguments juridiques, les déclarations publiques et les polémiques mis en avant par les compagnies de transport transforment l'enjeu migratoire en une question de coûts et de bénéfices ou de libre concurrence. Ainsi lors du procès impliquant KLM, les avocats de la compagnie ont argué du fait que la législation néerlandaise défavorisait KLM par rapport à d'autres compagnies. Les Etats eux-mêmes rentrent dans cette logique. Aux quatre compagnies européennes qui menaçaient de déposer une plainte à la Commission européenne des droits de l'homme en 1990 pour contester le rôle de garde-frontières que le Royaume-Uni leur imposait, le ministre de l'Intérieur britannique répondit qu'il leur retirerait leur droit d'atterrissage ${ }^{15}$.

Les autres critiques des sanctions qui sont avancées portent sur le fait que le personnel des compagnies n'a pas à accomplir ces missions. Certains syndicats comme la CFDT en France pensent qu'il faudrait saisir l'OIT pour établir une clause de liberté de conscience aux employés qui ne veulent pas participer aux contrôles. Là encore, le débat ne tourne pas autour de l'efficacité de cette politique ou de ses effets.

Le cas de l'immigration entre la France et le Royaume-Uni revêt les mêmes caractéristiques. Eurotunnel et les compagnies de transport routier qui menacent de ne pas payer les amendes et ont entamé des recours en justice évoquent les pertes commerciales subies ${ }^{16}$. Seules quelques organisations comme le IRU (International Road Transport Union) et les sections des grands syndicats comme la CGT et la CFDT ${ }^{17}$ ont dit vouloir que soit respecté le «droit international humanitaire $»^{18}$. Quand Eurotunnel a demandé en septembre 2001 puis en janvier 2002 au tribunal administratif de Lille la fermeture immédiate du camp de Sangatte, ils ont invoqué des millions de pertes ${ }^{19}$. Si ces préoccupations sont conformes aux objectifs de l'entreprise, l'aspect humanitaire de la crise à Calais semble oblitéré. Le gouvernement français, en particulier le ministre communiste des transports, qui s'est ému de la situation des chauffeurs de camion emprisonnés au Royaume-Uni ${ }^{20}$ n'a pas remis en cause la législation britannique la qualifiant au contraire de trop attirante pour les candidats à l'asile ${ }^{21}$. L'énergie et les investissements considérables déployés autour de la gestion de la situation transManche et des incidents diplomatiques qui s'en sont ensuivis montrent d'ailleurs s'il est 
besoin que le calcul des pouvoirs publics qui consistent à laisser les agents privés gérer la situation est un échec ${ }^{22}$.

Effets pervers : des flux criminalisés et réorientés

Efficacité ou effets pervers? Comment mesurer l'efficacité du système par rapport aux objectifs officiels (la lutte contre l'immigration illégale) et officieux (la baisse des demandes d'asile)? Les quelques rapports des services concernés se bornent à comptabiliser les amendes (par compagnie, par aéroport d'origine et de destination) afin de distribuer bons points et avertissements et de corréler l'entrée en vigueur du système avec le nombre de demandes d'asile et d'étrangers refoulés à la frontière. Il semble que les lois contre les transporteurs ont de toute façon donné lieu à très peu de procédures d'évaluation.

La première méthode n'apporte que peu d'éléments, les ministères le reconnaissent eux-mêmes. Le Conseil des ministres européens Justice et Affaires intérieures a d'ailleurs étudié une proposition française qui voulait inclure dans le formulaire du «dispositif d'alerte précoce» des données sur «la mise en conformité des transporteurs » que pourrait exploiter aussi le CIREFI. Le nombre d'amendes assez important au début baisse une fois que les compagnies ont eu le temps de s'organiser puis remonte une fois que les migrants eux-mêmes se sont organisés en conséquence. Sauf en Grèce, le nombre d'infractions tend à rester stable ou à augmenter ${ }^{23}$. Mais il y a d'autres variables qui rendent difficiles l'interprétation des données qui elles-mêmes prêtent à confusion, en particulier l'application de la réglementation par la police des frontières.

C'est d'autant plus le cas lorsqu'il existe des accords tacites ou écrits entre les autorités et les compagnies, selon lesquels les amendes ne seront pas payées ou les procèsverbaux non remplis si la compagnie respecte certains engagements ou parvient à descendre au-dessus d'un certain nombre d'infractions fixé à l'avance. Il est à noter que les amendes n'apparaissent pas dans les comptes de certaines entreprises comme Air France et que les données fournies par les ministères sont partielles et périmées et ne précisent pas quel montant a réellement été acquitté par les compagnies ${ }^{24}$. D'après les entretiens que nous avons pu effectuer, il semble néanmoins que le nombre de personnes que l'on ne laisse pas embarquer est assez similaire d'une compagnie à l'autre et tourne autour de 4000 à 5000 par an et par compagnie ${ }^{25}$. British Airways a publié le chiffre de 5568 passagers refusés à l'embarquement en $1995^{26}$. Les chiffres pour KLM oscillent autour de 4000 par an, on parle de plusieurs centaines de refus par mois à Air France. Par exemple, en août 2001, Air France a dit avoir refusé l'embarquement à 813 passagers et 89 de ses passagers ont été non-admis dans les pays de destination. 350 de ces refus ont eu lieu dans les escales africaines d'Air France à destination de Roissy et 60 passagers n'ont pas été admis sur le territoire ${ }^{27}$.

Si l'on étudie l'effet des sanctions contre les transporteurs sur les demandes d'asile, là encore il faut faire preuve de prudence. Il semble par exemple en Grande-Bretagne, que les demandes d'asile aux aéroports soient moins nombreuses mais elles sont plus que compensées par les demandes faites par des personnes déjà sur le sol britannique en possession de visas touristique ou étudiant ou par des overstayers. La Grande-Bretagne reste une destination privilégiée mais les candidats à l'asile essaient de passer par l'Eurotunnel ou dans des ferries à Calais.

Nous en venons aux conséquences non anticipées ou aux effets pervers des lois contre les transporteurs. Les demandeurs d'asile ont dû avoir recours à des personnes qui 
pouvaient leur procurer des faux documents de voyage (parfois détruits lors du vol) ou de vrais documents que les passagers restituaient après le contrôle effectué avant l'embarquement par les compagnies. D'une manière plus générale, les migrants potentiels arrivent de plus en plus par voie terrestre, après des périples autour du monde pour éviter les risques des contrôles aux aéroports. Là encore, ils ont besoin de faire affaire avec des trafiquants ${ }^{28}$.

Comme d'autres mesures de contrôle migratoire, la cooptation d'acteurs privés a limité les possibilités d'arriver sur le territoire des pays d'accueil. Mais, les pressions migratoires demeurent et les catastrophes humanitaires se succèdent. Par conséquent, on observe une augmentation de la criminalisation du phénomène migratoire. Les études existantes ont en effet montré que, pour surmonter les obstacles que constituent les nouvelles mesures de contrôle et de restriction du droit d'asile, les personnes fuyant les guerres civiles ou autres persécutions ont recours à des passeurs pour arriver dans l'Union européenne ${ }^{29}$ comme cela a pu être le cas pour les réfugiés Kosovars qui payaient entre 2000 euros et 8000 euros pour être amenés dans un pays de $l^{\prime}$ Union $^{30}$. Loin de s'interroger sur les conséquences de ce phénomène pour les migrants, les demandeurs d'asile et pour les sociétés où opèrent les trafiquants, les ministères invoquent l'immigration illégale pour justifier la pérennité des instruments de contrôle migratoire.

Des effets sur les droits des individus

Comme nous l'avons évoqué ci-dessus, les sanctions contre les transporteurs visent surtout à empêcher l'arrivée de demandeurs d'asile qui auparavant pouvaient tenter de quitter leur pays même sans visa ou passeport. L'organisation de la vérification des documents contribue en pratique à rendre difficile l'accès aux avions des groupes les plus susceptibles d'être persécutés ${ }^{31}$. En effet, les personnels des compagnies et ceux des agences de sécurité sous-traitées au départ ont tendance à appartenir à l'ethnie dominante. Il n'y a, par exemple, pas de Kurdes qui travaillent à Air France à Istanbul ou de Tamouls qui travaillent pour British Airways dans le pays de départ ${ }^{32}$. Le procédures de recours auprès des compagnies n'existant pas.

Mais le droit à l'asile n'est pas le seul des droits individuels qui risque d'être violé dans les pratiques des compagnies de transport. Une entreprise privée n'a pas les mêmes objectifs, les mêmes critères de succès qu'une agence de l'Etat. C'est là que la délégation à des agents privés a des conséquences non négligeables. En effet, les transporteurs suivent une logique commerciale dans un domaine où la compétition est féroce. Comme le rappelle Antonio Cruz, perdre un créneau horaire à cause de la photocopie de documents de voyage est extrêmement onéreux. C'est pourquoi on peut penser que des critères tels que le respect des droits de l'homme ne seront pas prioritaires ${ }^{33}$ ou qu'il y aura des cas de discrimination raciale, condamnée par la Convention européenne des droits de l'Homme et désormais aussi réprouvée par les Etats membres de l'Union lors de prestations de services par la directive européenne 2000/43 du 29 juin 2000 (article $3.1 \mathrm{~h}$ ).

En effet, les responsables de la sécurité des compagnies aériennes prônent la technique du profiling - c'est-à-dire la sélection de certains individus pour des contrôles plus poussés - plutôt que de retarder le départ du vol en soumettant tout le monde au même traitement. Le vice président de KLM chargé de la sécurité, Teun Platenkamp, préfère les "stratégies de sécurité ciblées" (focused security strategies) aux "approches compréhensives »(blanket approaches) dans les aéroports en arguant du fait qu'une 
mesure qui vise tout le monde qui n'ajouterait que 30 secondes par passager entraînerait « 3 heures et demie de plus de temps de traitement par vol » ce qu'il considère comme un énorme gâchis de ressources ${ }^{34}$.

Bien que le profiling ait été développé dans les années 1970 dans le cadre de la lutte antiterroriste, il semble que la même technique puisse être employée pour identifier les personnes «à risque migratoire». Les personnels des compagnies interrogées m'ont fait part de consignes qui visaient à déceler des comportements suspects lors de l'enregistrement (nervosité, habillement, air de chercher quelqu'un, etc.) qui s'approchent des critères d'identification d'autres types de "personnes à risque " (terroristes par exemple). Il est cependant impossible de savoir combien de personnes parmi les centaines de personnes non admises à embarquer chaque mois par chacune des compagnies étudiées l'ont été sur leur comportement.

Lorsque la réglementation sur les sanctions contre les transporteurs forcent les compagnies à fournir la photocopie des passeports des passagers pour prouver qu'ils les possédaient au départ s'il les détruit en vol et ainsi démontrer leur bonne foi, pour gagner du temps, la solution est de ne photocopier que les passeports des personnes «suspectes » et donc d'avoir recours au profiling. British Airways a d'ailleurs dû régler une somme importante pour éviter une condamnation devant les tribunaux dans une affaire où British Airways avait fait la photocopie des passeports d'un passager noir né en Grande-Bretagne et de sa famille qui l'avaient accusé de discrimination raciale ${ }^{35}$. La compagnie aérienne TAP a quant à elle été poursuivie pour discrimination raciale à la suite de la divulgation d'une note interne qui demandait au personnel de photocopier les passeports de tous les «noirs et métis » ${ }^{36}$.

C'est ainsi qu'on se rend compte que le système des sanctions transfère non seulement le coût des contrôles aux compagnies mais la responsabilité légale. Dans les affaires concernant TAP mais aussi dans les procédures civiles au Royaume-Uni entamées par des ressortissants kenyans et sri lankais contre les compagnies qui ne les avait pas acheminés ${ }^{37}$, on peut se demander si ce ne sont pas les lois contre les transporteurs qui ont encouragé les pratiques discriminatoires des agents privés.

Il faut à ce propos rappeler que la jurisprudence européenne dans les cas où des agents non-étatiques agissent hors des frontières mais en conformité avec les lois d'un Etat, cet Etat peut également être tenu pour responsable des infractions en même temps que le tiers parti. Dans Amuur v. France, la Cour européenne des droits de l'homme a souligné que bien que les plaignants, détenus en zone de transit internationale n'était pas selon la législation française sur l'immigration sur le territoire, ils étaient bien soumis à la loi française et la France devait respecter la Convention ${ }^{38}$. En outre, dans Mussele v. Belgium ${ }^{39}$, la Cour a conclu qu'un Etat ne pouvait échapper à ses obligations définies par la Convention en les déléguant à d'autres ${ }^{40}$.

Dans d'autres cas, connus sous le nom de "transit interrompu ", les passagers sont débarqués avant leur destination finale. Ce phénomène est assez répandu pour faire l'objet de dispositions législatives et réglementaires. Ainsi, en France, l'article 35 quarter, paragraphe VII de l'ordonnance du 2 novembre 1945 précise que peut être maintenu en zone d'attente un "étranger qui se trouve en transit dans une gare, un port ou un aéroport si l'entreprise de transport qui devait l'acheminer dans le pays de destination ultérieure refuse de l'embarquer ». Comme le note François Julien-Laferrière, la circulaire attenante du 9 juillet 1992 souligne que ces étrangers ne font pas l'objet d'une décision de non-admission : «ils ne bénéficient d'aucune garantie et peuvent être renvoyés sans délai, 
sans formalité, sans qu'il en reste aucune trace. Aucune décision ne leur est notifiée, puisqu'il $n^{\prime} e n$ est pris aucune ${ }^{41}$. Les transporteurs, par peur des sanctions, placent leurs passagers dans une situation extrêmement floue et précaire d'un point de vue juridique.

Des passagers suspectés par le personnel aérien de ne pas avoir les documents de voyage requis ont également été retenus dans les zones internationales, comme c'est le cas en Russie. Comme le souligne Frances Nicholson, depuis 1992, la zone de transit Sheremetyevo 2 de l'aéroport de Moscou « a retenu jusqu'à vingt passagers en même temps, dont des réfugiés qui se rendaient dans les Etats d'Europe de l'Ouest [et] qui n'ont pas été autorisé à embarquer par des compagnies qui voulaient éviter de payer des amendes $»^{42}$. Or ces passagers ne pouvaient pas demander l'asile à Sheremetyevo $2^{43}$.

Le rôle des agents privés dans le contrôle de l'immigration a des antécédents historiques et dans un pays comme les Etats-Unis les sanctions pécuniaires existent depuis presque un demi-siècle. Dans les années 1980, ce rôle a connu un renouveau important puisque les gouvernements désiraient empêcher l'arrivée d'étrangers non sollicités mais devaient prendre en compte un cadre juridique respectueux des droits de l'homme. Il semble que, depuis lors, l'implication d'entreprises privées comme les compagnies aériennes soit devenue la norme, un instrument de politique publique courant, généralisé comme si cela avait toujours été une évidence. Pourtant, l'impact de ces mesures est difficile à évaluer - et ne l'a jamais été de façon systématique par les autorités. Elles posent un certain nombre de problèmes vis à vis du droit international en matière d'asile et ont des effets pervers non négligeables. A qui profitent ces mesures? Aux agences de l'Etat qui restent maîtres du jeu, transfèrent un certain nombre de coûts au privé et encaissent les amendes. Aux agences de sécurité et aux anciens fonctionnaires reconvertis. Aux passeurs et aux producteurs de faux documents.

Pourtant, le système continue de s'étendre à d'autres pays (de l'Europe de l'Est au Nigéria). Les pays d'Europe Centrale et de l'Est par exemple se sont engagés à Berlin en 1994 à mener "une intervention conséquente à l'égard des entreprises de transport aérien et maritime qui transportent des étrangers qui ne sont pas en possession des documents nécessaires $»^{44}$. Avec l'entrée en force du traité d'Amsterdam, les pays candidats à l'accession doivent se conformer à l'acquis Schengen donc à l'article 26. Les lois deviennent plus strictes: en 1990, les amendes sont passées de \$1000 à \$3000 aux Etats-Unis, en 1993, de FF 5000 à 10000 en France, au Royaume-Uni en 1991 de $£ 1000$ à 2000. Avec la nouvelle directive, la plupart des pays vont devoir augmenter les amendes. La France compte ainsi passer à 30000 francs, vers le haut de la fourchette établie dans la directive européenne (de 5000 euros).

De récents développements indiquent qu'à la responsabilité du secteur privé dans la politique migratoire s'ajoute celle des individus qui eux aussi paieront les coûts de cette politique. C'est ainsi qu'un ressortissant belge détenu dans le centre de détention de Woomera en plein désert australien pour situation irrégulière s'est vu remettre une facture du gouvernement australien lors de son expulsion le 23 novembre 2001 lui demandant de régler sa note : 1000 euros de billet d'avion mais aussi 35 euros par jour pour son séjour dans le camp géré par une entreprise privée américaine spécialisée dans la construction des prisons, la Wackenhut Corrections Corporation ${ }^{45}$. De façon moins anecdotique, aux Etats-Unis, un forfait de \$ 6 («User fee ») est imposé à chaque passager arrivant de l'étranger et reversé à l'INS pour couvrir les frais assumés par 
l'INS pour la rétention des étrangers qui seront reconduits à la frontière. Tous participent même à leur insu - les touristes connaissent-ils le "User fee statute » ? - à la politique de contrôle migratoire.

\section{NOTES}

1.. British Airways a eu par exemple des problèmes avec les trafiquants sur les lignes allant au Canada et n'a pas signé de protocole d'entente avec Immigration Canada comme c'est le cas de Lufthansa qui est satisfait du protocole et sûr de pouvoir respecter les quotas d'infractions grâce entre autre au travail de l'agence de sécurité AVS dans les aéroports allemands qui recrute chez les anciens de la police des frontières (BGS).

2. . En mai 1998, trois ans après l'entrée en vigueur de la loi, la compagnie avait déjà dû payer 4500 amendes de 5000 florins à Schiphol.

3. . Avant qu'elle ne soit reprise sous l'enseigne du groupe Air France, UTA avait déjà des sous-traitants locaux qu'Air France a continué à employer.

4. . Marx, Reinhard, « Analyse du traitement réservé au requérants d'asile dans les aéroports allemands » in ANAFE, Frontières des droits - frontières du droit, Paris, L'Harmattan, 1993. Cf. également Frankfurter Rundshau, 5 septembre 1990.

5. . Document 96/622/JAI, « Position commune du 25 octobre 1996 définie par le Conseil sur la base de l'article K.3 paragraphe 2 point a) du traité sur l'Union européenne, relative aux missions d'assistance et d'information effectuées en amont de la frontière ", Journal officiel L281/1, 31.10.1996.

6. . Note de la Présidence française du 4 juillet 2000 sur un «plan d'action pour améliorer le contrôle de l'immigration ", Conseil de l'Union, Bruxelles, DOC 10017/00.

7.. Dans le cas de l'immigration trans-Manche où les transporteurs s'exposent à une amende de $€ 2000$ par passager en situation irrégulière, le même phénomène s'est produit puisque l'accord franco-britannique de l'automne 2001 prévoit l'envoi de cinquante officiers de l'immigration britanniques du côté français de l'Eurotunnel pour aider la compagnie à empêcher que des étrangers arrivent en Angleterre en empruntant le train ou le tunnel.

8. . «Conclusions orales tirées par Daniel Vaillant 》, neuvième conférence des ambassadeurs, "problématique sécurité et immigration », Paris, 28 août 2001. 9. . Le porte-parole d'Eurotunnel a évoqué le besoin d'avoir recours à « une pensée tactique » et le Général a lui évoqué ses compétences dans le « déploiement de la main d'œuvre ». «Ex-general to police Channel tunnel », CNN, 9 septembre 2001. URL : http://europe.cnn.com/2001/WORLD/europe/09/09/tunnel.asylum/index.html 10. . Platenkamp, Teun, « The Benefits of Focused Security strategies over Blanket Security Policies ", Communication du 14 janvier 1997 au colloque international « aviation safety and security » de l'université George Washington, Washington, DC, 1997.

11. Entretien avec un membre du personnel de KLM, mai 2001. 
12. . Dobbin, Frank, « Is America becoming more exceptional ? The privatization of social citizenship rights since $1960 »$ in Bo Rothstein et Sven Steinmo (dirs), Structuring the Welfare State : Political Institutions and Policy Change, New York, Palgrave, 2002. 13. . L'ECAC a un projet pilote en la matière sous l'auspice du groupe de travail « Facilitation », ECFALIS, ECAC facilitation information system on illegal immigration. Les statuts ont été modifiés le 14 décembre 2000 pour inclure une mise à jour de la gestion des passagers inadmissibles (paragraphe 2.3, DOC 30).

14. . Le plus récent est une conséquence du 11 septembre et se nomme Global Aviation Security Action Group (GASAG). Le responsable de KLM susmentionné y est impliqué. Cf. et son entretien sur le 11 septembre dans Edwin van Rij, « veilig door de lucht » in Op de Bok, octobre 2001 : http://www.vnv-dalpa.nl/opdebok/2001/20011002.html 15. . Daily Telegraph, 21 juin 1990.

16. . Cf. également le document de la SNCF intitulé « Position de la SNCF sur l'immigration et l'émigration vers la Grande-Bretagne. « La SNCF étant victime elle aussi de cette situation et subissant de plein fouet les conséquences (pertes financières causées par les dégradations, les retards, (...) et risques importants au niveau de la sécurité des personnes et des circulations » (p. 1). Annexe 3 du rapport du Conseil National Transports, L'immigration clandestine dans les transports, rapporteurs généraux : Pierre Bourgeois et Christine Murret-Labarthe, Paris, CNT. Il est consultable sur le site http://www.cnt.fr/rapports/Immigration_clandestine_ANNEXE3.pdf 17. . Cf. par exemple l'article intitulé « ni flics, ni passeurs » in Journal de la Fédération générale des transports et de l'équipement, Magazine CFDT n 13, 2ème semestre 2001. 18. . Cf. le communiqué de presse 648 de l'IRU du 6 février 2002, « IRU welcomes decision to withdraw penalty for illegal entrants ».

19. . Reuters, 11 septembre 2001.

20. . Cf. la communication de Chantal Receveur, membre du cabinet de M. Gayssot au colloque « Frontières et zones d'attente » de l'ANAFE, Sénat français, 19-20 octobre 2001.

21. . Le gouvernement a plutôt coopéré avec les autorités du Royaume-Uni et encouragé les transporteurs à le faire. Cf. les circulaires du ministère de l'Intérieur des 20 et 31 juillet 2001 aux préfets et la lettre du 16 novembre 2000 (avec fiche de contrôle en annexe). Le gouvernement a par ailleurs traduit et répercuté la « checklist » établie par les services britanniques pour les transporteurs qui exige des contrôles très poussés des véhicules (contrôle $\mathrm{CO} 2$, etc.). Ce document est disponible sur le site du Conseil national des transports (http://www.cnt.fr/rapports/Immigration_clandestine_ANNEXE2.pdf)

22. . Depuis 2000, le nombre de morts dans le tunnel a augmenté à mesure que les personnes tentaient de le traverser à pied ou de monter sur les trains.

23. 0 . Ministère de l'Intérieur, op. cit.

24. . A titre informatif, le ministère de l'Intérieur français a indiqué pour 1998 avoir établi 2175 procès-verbaux et infligé 1603 amendes et pour les 9 premiers mois de 1999, 2192 procès-verbaux et 1172 amendes (chiffres communiqués lors de la réunion annuelle sur le fonctionnement des zones d'attente du 25 octobre 2000). Rappelons qu'entre 1993 et 1995, 3444 amendes ont été prononcées (ministère de l'Intérieur 1996). 25. . Entretiens auprès de personnel de KLM, d'Air France et de British Airways. 26. . Nicholson, F., op.cit., p. 598.

27. . De Roissy vers le Royaume-Uni et l'Irlande, on dénombre pour le mois d'août 2001, 150 refus d'embarquement, vers les Etats-Unis 187 et vers le Canada 100. Données 
transmises à la section Air France de la CFDT. Cf. la communication de François Cabrera, CFDT Air France, au colloque « Frontières et zones d'attente » de l'ANAFE, Sénat français, 19-20 octobre 2001.

28. . Kyle, David et Ziai Liang, « Migration Merchants : Human Smuggling from Ecuador and China » in V. Guiraudon et C. Joppke (dirs.), Controlling a New Migration World, Londres, Routledge, 2001, pp. 200-221.

29. . Morrisson, John, The Trafficking and Smuggling of Refugees : The End of European asylum Policy ?, Rapport pour le Haut Commissariat pour les réfugiés, Genève, HCR, 2000, pp. 24-26.

30. . Koslowski, Rey, « Personal Security and State Sovereignty in a Uniting Europe » in V. Guiraudon et C. Joppke (dirs.), op. cit., p.108.

31. . Le rapport de la Commission nationale consultative des droits de l'homme de juin 2001 sur l'asile en France commente ainsi la directive européenne sur les transporteurs : « Le risque manifeste existe de voir se créer dans les pays d'embarquement des situations de discrimination et de pré-jugement de la qualité de réfugié par le personnel des compagnies aériennes : une personne risquant d'être persécutée pourra difficilement convaincre l'agent de la compagnie qu'elle est en danger et que, malgré l'absence de passeport ou de visa, il faut la laisser fuir. Cela lui sera d'autant plus difficile si cet agent est de sa nationalité, voire membre d'une ethnie rivale, sans qu'existe, en outre, de garantie de sécurité vis à vis de la police locale ». Commission nationale consultative des droits de l'homme, L'asile en France. Etude préparative à l'avis de la CNCDH, rapporteur général : Jacques Ribs, Paris, CNCDH, 2001. 32. . Cf. la communication de François Cabrera, CFDT Air France, au colloque « Frontières et zones d'attente » de l'ANAFE, Sénat français, 19-20 octobre 2001. Entretien à la CFDT d'Air France, février 2002.

33. . Ainsi, en 1990, trois passagers tamouls qui n'étaient pas en règle ont été « kidnappés » par le personnel de bord de British Airways qui avaient peur d'être sanctionné et renvoyé sur Rome, (The Daily Telegraph, 7 juillet 1990).

34. . Platenkamp, T., op. cit., p. 1.

35. . «BA chief apologizes for copying of 'ethnic passport' » Financial Times, 11 novembre 1995 et «BA pays tens of thousands in racism claim », The Guardian, 9 octobre 1996.

36. . de Stoop, Chris, Vite, rentrez le linge ! L'Europe et l'expulsion des sans-papiers, Arles, Actes Sud, 1996, p .228.

37. . Nicholson, F., op.cit., pp. 586-634.

38. . ECHR 25 juin 1996 (17/1995/523/609), paragraphe 52.

39. . ECHR 27 octobre 1983 Series A, n ${ }^{\circ} 70$ (1983), paragraphe 29.

40. . Evert A. Alkema, ancien membre de la Commission européenne des droits de l'homme, a affirmé que si une plainte contre une personne y compris une compagnie de transport était incompatible avec l'article 25 de la Convention, le concept de Drittwirkung permet de déposer une plainte comme un Etat signataire si ce dernier est responsable de la violation des droits protégés par la Convention. A son avis, cela est " particularly relevant at a time when several governments are seeking to transfer to private corporations tasks formerly entrusted to public bodies» (1988, p. 40).

41. . Julien-Laferrière, François, « La situation des demandeurs d'asile dans les zones d'attente et les lieux de rétention administrative » in Cultures \& Conflits 23 , automne 1996, p.7.

42. . Nicholson, F., op.cit, pp. 598-9. 
43. . En outre, la coopération des compagnies aériennes quant il s'agit de renvoyer les non-admis sur le prochain vol en partance n'est pas anodine. Cela permet aux autorités de ne pas détenir les étrangers et de ne pas leur donner accès à l'aide juridique. En France, la loi permet aux non-admis de rester un jour franc mais en pratique cela n'est pas respecté (par exemple, en cochant à l'avance sur les formulaires la case qui indique qu'on y renonce). Aux Etats-Unis, une longue et difficile procédure d'éloignement est souvent évitée grâce aux « same-day removals » facilitées par la bonne volonté des compagnies.

44. . Déclaration de Berlin adoptée le 8 septembre 1994.

45. 5000 personnes détenues y ont entamé une grève de la faim le 17 janvier 2002 et des enfants se sont cousus la bouche en protestation des conditions du camp : forte chaleur, enfermement, manque d'hygiène et de nourriture. Cf. Frédéric Thérin, « Demandeurs d'asile, bouches cousues derrière les barbelés » in Le Monde, mardi 29 janvier 2002, p. 35.

INDEX

Mots-clés : contrôle, entrepreunariat, migration, mobilité, police à distance, privatisation de la sécurité 